\title{
Novel Microfluidic Structures for Wireless Passive Temperature Telemetry Medical Systems Using Radar Interrogation Techniques in Ka-Band
}

Sofiene Bouaziz, Franck Chebila, Anya Traille, Patrick Pons, Hervé Aubert, and Manos M. Tentzeris, Fellow, IEEE

\author{
(Invited Paper)
}

\begin{abstract}
We present a new miniaturized (below $1 \mathbf{m m}^{3}$ ) temperature sensor based on microfluidic technology and radar passive interrogation principles, which can be easily applied for temperature telemetry for medical applications. The chipless microsystem is made up of a planar-gap capacitor with a microchannel located in between its plates. The temperature-dependent expansion/shrinkage of the water inside the microchannel modifies in a monotonic way the liquid level across the capacitor. The resulting change in the effective permittivity modifies the capacitance value in a temperature-dependent way. The first prototypes of the temperature microsensor were micromachined and integrated with an antenna, while the ambient temperature was remotely measured using frequency-modulated continuous-wave (FMCW) radar interrogation principles at $29.75 \mathrm{GHz}$. Preliminary measurement results demonstrated a $0.4 \mathrm{dBm} /{ }^{\circ} \mathrm{C}$ sensitivity over a $9^{\circ} \mathrm{C}$ temperature range $\left(24^{\circ} \mathrm{C}-33^{\circ} \mathrm{C}\right)$.
\end{abstract}

Index Terms-Electromagnetic transduction, frequency-modulated continuous-wave (FMCW) radar, medical telemetry, microfluidic, passive and chipless sensor, remote sensing, temperature microsensor, wireless sensor.

\section{INTRODUCTION}

$\mathbf{E}$ ARLY temperature sensors have been based on the temperature-dependent volume expansion of liquids/solids quantified by their position within an enclosing container. This old technique is still in widespread use because it meets the needs of many common applications in a reliable and cost-effective manner [1], [2]. Nevertheless, wireless passive temperature sensors can be crucial in "rugged" medical and biotelemetry applications, such as the battery-less temperature monitoring of different patients throughout different locations of a hospital or the continuous monitoring of the storage temperature of bio-substances, while they can also be extremely effective in monitoring the health status of a large herd of animals (veterinary telemetry systems) without increasing farming costs.

Manuscript received October 16, 2012; revised December 18, 2012; accepted January 03 , 2013. Date of publication January 23, 2013; date of current version January $31,2013$.

S. Bouaziz, F. Chebila, A. Traille, P. Pons, and H. Aubert are with the LAASCNRS and University of Toulouse; UPS, INSA, INP, ISAE; 31077 Toulouse, France (e-mail: sbouaziz@laas.fr).

M. M. Tentzeris is with the School of ECE, Georgia Institute of Technology, Atlanta, GA 30332 USA.

Color versions of one or more of the figures in this letter are available online at http://ieeexplore.ieee.org.

Digital Object Identifier 10.1109/LAWP.2013.2242272
Over the years, several research efforts have been performed to satisfy this need, e.g., the use of surface acoustic wave (SAW)-based sensors [3], which are sensitive to surface contaminations (extreme attention is required during the packaging), and radio frequency identification (RFID)-based sensors [4] with a limited use due to their commonly short reading range for passive configurations. Consequently, a new generation of wireless passive sensors has emerged, in the past few years, based on electromagnetic transduction and radar interrogation [6], [7]. Previous works published by the authors have shown the feasibility of temperature sensor based on liquid expansion and radar passive interrogation [5]. It is shown that liquid metal can be used to dynamically alter the number of antenna elements or scatterers activated along a linear array configuration, thus allowing the "discretized" measurement of the temperature.

In this letter, we take advantage of the unique properties of the microfluidic technology: Dielectric or conductive fluids placed in the proximity of a microwave component can significantly change its scattering parameters. A new advanced miniaturized (volume less than $1 \mathrm{~mm}^{3}$ ) temperature-monitoring system combining microfluidic technology and wireless reading techniques and being easily integrable in biotelemetry systems is hereby presented. This temperature sensor allows continuous battery-less and wireless temperature measurement. The temperature-dependent volume expansion of water within a microfluidic channel is used to progressively change the effective permittivity of the medium between the plates of an underlying planar gap capacitor. Consequently, it modifies the capacitance value and the scattering parameters of the structure. The subsystem composed of the sensor and an antenna is then interrogated using frequency-modulated continuous-wave (FMCW) radar. In this way, the radar echo level increases or decreases with respect to the temperature.

In the following sections, the sensor design steps will be presented followed by electromagnetic simulation results, fabrication process, experimental setup description, and remote measurement of temperature using one millimeter-wave FMCW radar reader.

\section{Microfluidic Temperature Sensor Concept}

\section{A. Design}

The proposed wireless sensor relies on the development of microfluidic tunable capacitors for wireless temperature sensing 


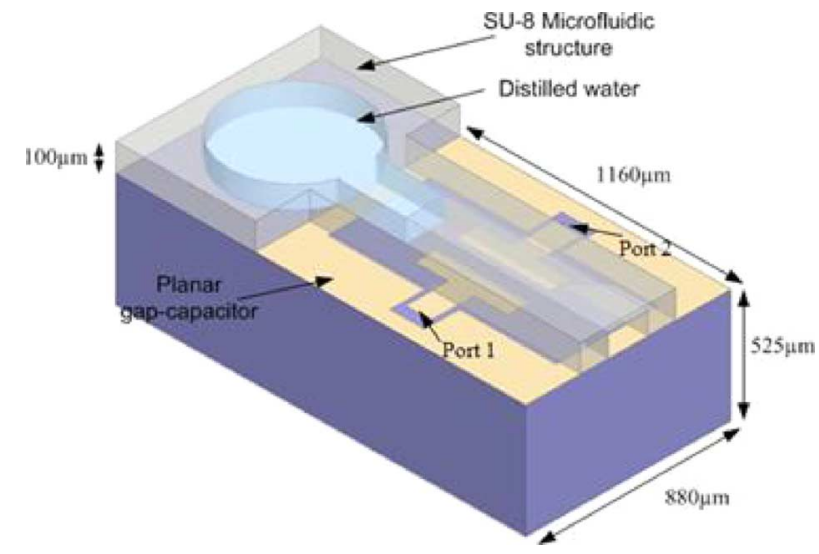

Fig. 1. 3-D schematic of the proposed microfluidic temperature microsensor: tunable microfluidic planar gap capacitor using temperature-dependent water volume expansion inside a microchannel.

using a temperature-dependent radar echo. The device is made up of a planar gap capacitor with a water-filled microchannel situated above the plates. The dielectric liquid progressively/gradually fills the channel due to its thermal expansion as the temperature increases. This rising level of high-permittivity liquid within the capacitor modifies the scattering parameters of the microfluidic structure.

The sensor structure is presented in Fig. 1 and optimized using full-wave electromagnetic simulations based on finite element method (HFSS). The two coplanar waveguides (CPWs) access lines are fabricated with a $100-\mathrm{nm}$ gold layer on a $525-\mu \mathrm{m}$ glass substrate. These CPWs present a characteristic impedance of $50 \Omega$ with a $110 \mu \mathrm{m}$ wide center conductor and $20 \mu \mathrm{m}$ wide spacing between the center conductor and the ground plane.

The planar gap capacitor dimensions were optimized to increase the capacitance sensitivity to the water-filling level of the channel. Moreover, the influence of parasitic capacitances existing between capacitor plates and ground plane was reduced. The planar capacitor plates have the following dimensions: $90 \mu \mathrm{m}$ width, $400 \mu \mathrm{m}$ length, and $90 \mu \mathrm{m}$ spacing between plates. A miniaturized $\left(170 \times 100 \mu \mathrm{m}^{2}\right.$ cross section) SU-8 microchannel, filled with water, was placed over the coplanar capacitor along the plates. Electromagnetic (EM) simulation results show a monotonic $8-\mathrm{dB}$ variation of the reflection coefficient $S_{11}$ with respect to the filling level with water of the gap between the capacitor plates. The tunable capacitance is varying from $9 \mathrm{fF}$ for an empty gap to $145 \mathrm{fF}$ when the gap is completely filled with water.

Meanwhile, a large circular tank connected to the microchannel is needed to provide the required liquid displacement (equal to $L$ ) for a given temperature range, just as it is made in standard thermometers. We use a tunable temperature range of $10^{\circ} \mathrm{C}$ (between $25^{\circ} \mathrm{C}$ and $35^{\circ} \mathrm{C}$ ) where the temperature-dependent volume expansion of water is quasi-linear $\left(257 \mathrm{ppm} /{ }^{\circ} \mathrm{C}\right.$ at $\left.25^{\circ} \mathrm{C}\right)$. In addition, the maximum measurable temperature range is limited by the low-temperature negative expansion coefficient and high-temperature evaporation of water. Consequently for commercial application, it can be suggested to use other dielectric fluid with similar performances. Therefore, the structure presents a tunable temperature range $(\Delta T)$ that depends on the liquid thermal expansion coefficient $(\alpha)$, tank volume $\left(V_{T}\right)$, microchannel section $\left(S_{\mathrm{c}}\right)$, and the length of the capacitor plates ( $L=400 \mu \mathrm{m}$ here) by the following relation:

$$
\Delta T=\frac{L S_{\mathrm{c}}}{\alpha} \frac{1}{V_{T}} .
$$

To increase the temperature range, one can increase the channel section, decrease the tank volume, use liquid with lower dilation coefficient, or design a new capacitor with enlarged length. Consequently, it is possible to enlarge the full-scale temperature range $\Delta T$ at least by a factor 10 .

\section{B. Wireless Passive Temperature Remote Sensing Principle}

The temperature sensor structure is designed to be integrated with directive planar antenna geometries such as a linear planar dipole-antenna array. Indeed, a theoretical study proves that the temperature-dependent cell utilized as the load of a planar dipole antenna reduces the surface current and consequently modifies its radar echo level. Simulation results, done with metallic liquid, show a full-scale radar echo range of $13 \mathrm{dBsm}$ [5].

In this letter, the temperature sensor, being connected from one port via a $50-\Omega$ coaxial cable of physical length $L=1 \mathrm{~m}$ to a broadband horn antenna, represents its load. A more compact design option would be to use a miniaturized microstrip delay line [8]. The other port of the sensing device is loaded by $50 \Omega$. The subsystem forms a completely passive and tunable $\mathrm{RC}$ sensing scheme. The temperature-dependent change of the sensor input impedance modifies the impedance loading condition of the antenna input and varies the antenna-sensor echo level. The echo level is here measured by using a radar reader. Using this configuration, at ambient temperature, two distinct backscattered signal peaks are expected in the beat frequency signal spectrum [8]. First echo, at a specific beat frequency $f_{\text {str }}$, represents the structural scattering mode, which depends on the antenna shape (geometry and material). Second echo, at a different beat frequency $f_{\mathrm{ant}}$, represents the antenna scattering mode and depends mostly on the impedance loading condition of the antenna [9]. This echo level was used in this work to measure the temperature dependent variation of the sensor capacitance. The coaxial delay line length is chosen to be 5 times the minimum resolution of the radar to be long enough to keep the antenna scattering mode and structural scattering mode separate, but also short enough to reduce losses.

The maximum measurement range of the wireless sensor is limited to the minimum detectable antenna backscattered power level that exceeds the mean noise level. On the other hand, the antenna-sensor backscattered power level corresponds to a temperature-dependent tunable physical function of its input reflection parameters. Consequently, the radar echo level measurement range is maximized by designing the microfluidic sensor structure input impedance to vary from open circuit to $50 \Omega$, regardless of the required liquid volume.

\section{Fabrication Process of the Temperature Cell}

The fabrication process flow, shown in Fig. 2, requires three masks. During the technological process, a $\mathrm{Ti} / \mathrm{Cu}$ $(0.1 \mu \mathrm{m} / 0.1 \mu \mathrm{m})$ seed layer was first sputtered on both sides of 


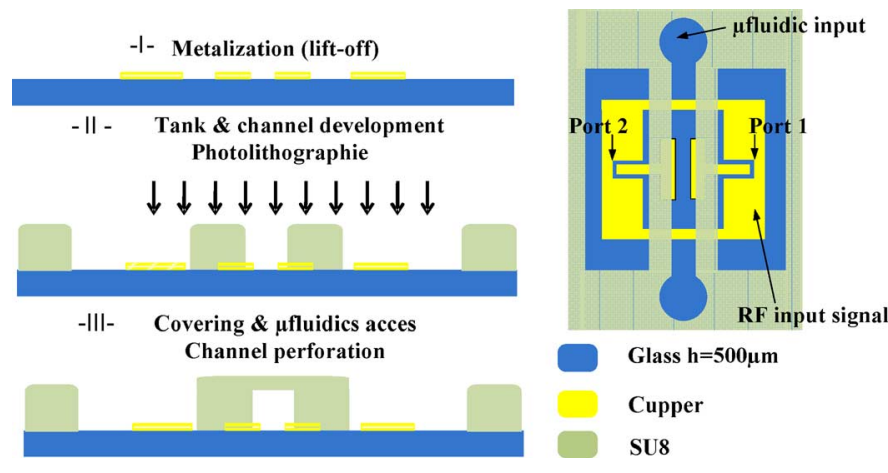

Fig. 2. (left) Fabrication process flow. (right) Top view of the structure.

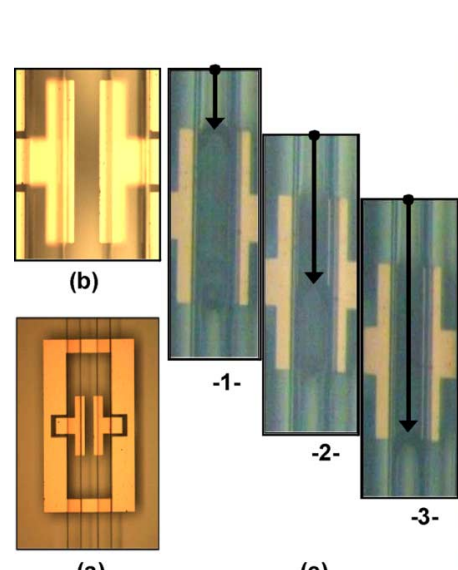

(a)

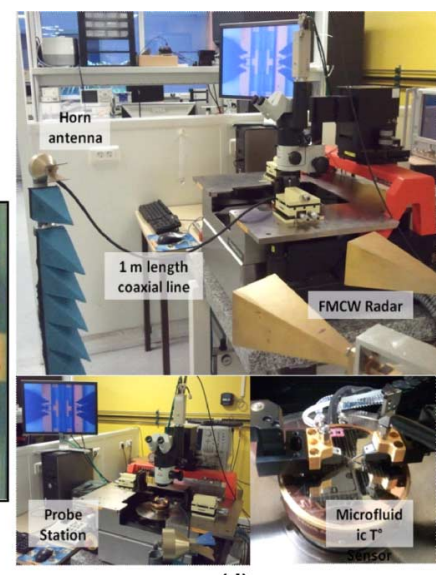

(d)
Fig. 3. (a) Fabricated temperature sensor cell. (b) Sensitive part. (c) Microchannel filled at (c1) $0 \%$, (c2) $50 \%$, and (c3) $100 \%$ with water. The arrows indicate the direction of water thermal expansion inside the channel. (d) On wafer $\mathrm{RF} /$ temperature measurement bench.

a 525- $\mu \mathrm{m}$-thick glass substrate. In this way, the planar capacitor structure with a ground plane on the backside is made using the lift-off method. In order to increase the adhesion of the SU-8 on glass or silicon wafers, an adhesion promoter can be used. Afterwards, channels and tanks were micromachined using photolithography on $100-\mu \mathrm{m}$ SU-8.

A photosensitive SU-8 dry film, $50 \mu \mathrm{m}$ in thickness, is then laminated and patterned on top of the channel to close the structure and fabricate the fluidic access, as reported in [10]. In that way, two holes were perforated in the cover. One hole is sealed from the tank side with a square piece of glass and double-sided adhesive. The second hole is used to fill the structure and is placed in a container filled with distilled water and placed under vacuum. The fluid is then forced into the SU-8 microstructure using pressure [11]. Fig. 3(a) presents the fabricated sensor, while the temperature-sensitive part is in Fig. 3(b). However, other dielectric liquid with similar performance can be used in this application. Water volume expansion tests are made with respect to temperature inside microstructure of SU-8. Fig. 3(c) shows the positions of the water level inside the channel for various temperatures.

The results obtained from this measurement are displayed in Table I. A measured linear thermal expansion of $43 \mu \mathrm{m} /{ }^{\circ} \mathrm{C}$ is observed in the range of $24.2^{\circ} \mathrm{C}$ to $33.2^{\circ} \mathrm{C}$. Measured accuracy is estimated at $\pm 3 \mu \mathrm{m} . T_{0}$ denotes the measured temperature for

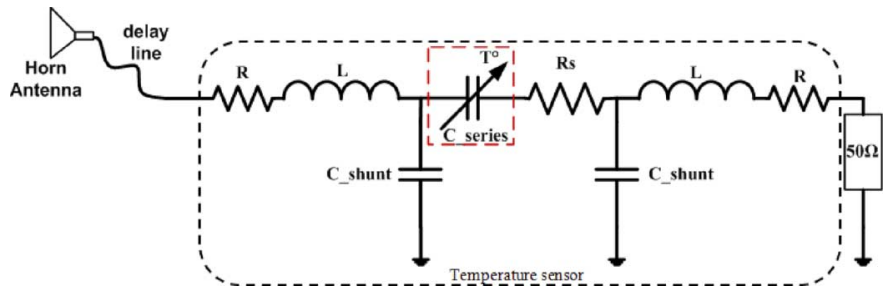

Fig. 4. Equivalent circuit of the microsystem.

TABLE I

THERMAL EXPANSION $\Delta L c$ OF WATER INSIDE MiCROSTRUCTURE OF SU-8

\begin{tabular}{|c|c|c|c|}
\hline Filling rate of the channel & $\mathrm{T}\left({ }^{\circ} \mathrm{C}\right)$ & $\mathrm{T}-\mathrm{To}\left({ }^{\circ} \mathrm{C}\right)$ & $\Delta \mathrm{Lc}(\mu \mathrm{m})$ \\
\hline $0 \%$ & 24,2 & 0 & 0 \\
\hline $21 \%$ & 26,1 & 1,9 & 85 \\
\hline $39 \%$ & 27,7 & 3,5 & 158 \\
\hline $54,5 \%$ & 29,1 & 4,9 & 229 \\
\hline $75,5 \%$ & 31 & 6,8 & 305 \\
\hline $100 \%$ & 33,2 & 9 & 387 \\
\hline
\end{tabular}

an empty gap between the capacitor plates $(0 \%$ of filling rate of the channel), and $\Delta L c$ is the displacement (in micrometers) of the water front inside the microchannel.

\section{Readout Method AND Results}

\section{A. Temperature-Dependent Capacitance Variation Measurement}

A probe station operating up to $40 \mathrm{GHz}$ and an Anritsu vector network analyzer (VNA) were used for the capacitance measurement of the temperature cell. The RF/Temperature measurement bench is composed by a classical RF measurement bench with a heating chuck [Fig. 3(d)]. For all the following measurements, the microfluidic capacitor temperature sensor is terminated with a 50- $\Omega$ load at one port and connected to a coaxial delay line of $1 \mathrm{~m}$ length (sufficient length to separate the structural-scattering mode from the antenna-scattering mode) from the other port. The termination of the coaxial delay line is connected to the VNA.

The equivalent electrical circuit of the temperature sensor shown in Fig. 4 is extracted considering different parasitic components as well as the RF-probes and setup effects. Then, the $S$-parameters calculated from the equivalent circuit are fitted with the measured ones. Thereby, the measured values of the variable capacitance $C_{-}$series, which represents the sensitive component of the temperature sensor, labeled by a red-dotted framework in Fig. 4, are extracted. The measurement results are varying linearly from $C_{\_}$series $=18 \mathrm{fF}$ for an empty channel at $24.2^{\circ} \mathrm{C}$ to $C_{-}$series $=143 \mathrm{fF}$ when the channel is completely filled at $33.2^{\circ} \mathrm{C}$. Unchanged values of parasitic elements representing the two CPWs accesses $(L=0.1 \mathrm{nH}$ and $R=10.2 \Omega$ ) are added from both sides of the variable capacitance $C_{-}$series . The parasitic capacitance existing between capacitor plates and the ground plane is represented by a capacitance $C_{\_}$shunt $=$ $23 \mathrm{fF}$. An additional resistance $R=37 \Omega$ is introduced in series with $C_{-}$series, which models the losses added by both SU-8 resin forming the channel and the dielectric liquid. The measured temperature-dependent capacitance extracted values are 


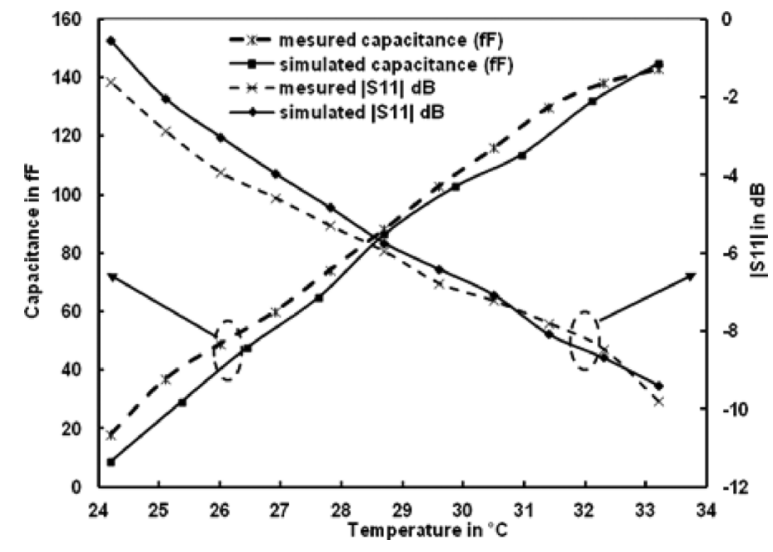

Fig. 5. Measured and simulated series capacitance and reflection coefficient versus applied temperature at $29.75 \mathrm{GHz}$.

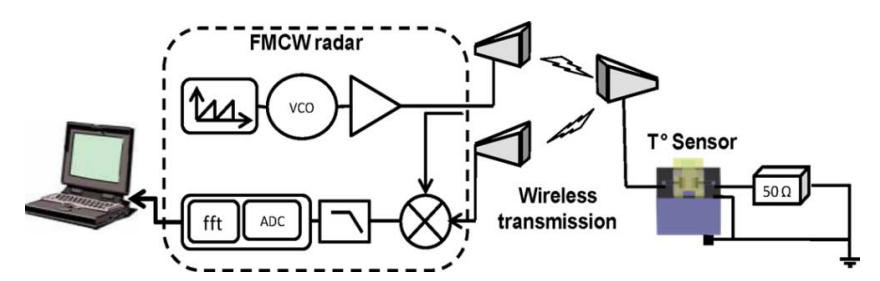

Fig. 6. Experimental setup using FMCW radar reader.

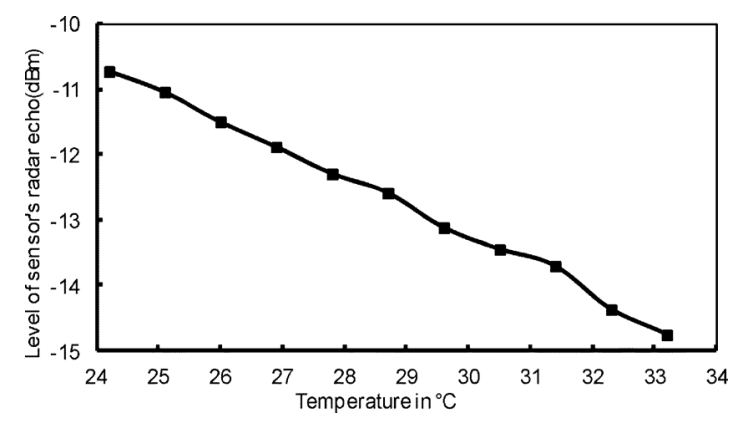

Fig. 7. Measured radar echo level versus temperature.

compared to the simulation results for the carrier frequency of the FMCW radar $\left(f_{0}=29.75 \mathrm{GHz}\right)$ in Fig. 5 .

The measured sensitivity corresponds to $13 \mathrm{fF} /{ }^{\circ} \mathrm{C}$ when associating the filling level with the temperature expansion of water. A small constant offset is observed in the difference between simulation and measurement results, particularly for lower measured temperature as a consequence of microfabrication tolerances.

\section{B. Temperature Remote Sensing Using FMCW Radar Reader}

The EM sensor interrogation technique using FMCW radar [9] allows a real-time remote sensing at a long reading range in addition to a low-cost implementation with a principle shown in Fig. 6. The transmitted chirp signal has a bandwidth of $650 \mathrm{MHz}$ and a 1-kHz period of frequency modulation of the radar carrier frequency $f_{0}=29.75 \mathrm{GHz}$. The measurement results verify that the proposed chipless temperature sensor features a good performance and allows the wireless passive temperature sensing. Following the configuration of paragraph
Section II-B, a 40-GHz horn antenna with high gain $(10 \mathrm{~dB})$ is connected to the temperature cell as the target for the FMCW radar. The target antenna was placed at $2 \mathrm{~m}$ and adjusted to be aligned with the $\mathrm{Tx} / \mathrm{Rx}$ antennas of the radar. Dual- or circularly polarized antennas could be used for avoiding undesirable impact of antennas misalignment on the RCS measurement. The change in the applied temperature causes the variation of the input impedance of the microfluidic capacitor. As expected, a quasi-linear decrease in radar echo level is measured at beat frequency $f_{\text {ant }}=20.5 \mathrm{kHz}$ as shown in Fig. 7. The measured sensitivity is $\sim 0.4 \mathrm{dBm} /{ }^{\circ} \mathrm{C}$ over a $9^{\circ} \mathrm{C}$ temperature range $\left(24^{\circ} \mathrm{C}-33^{\circ} \mathrm{C}\right)$.

\section{CONCLUSION}

The concept of a new miniaturized temperature sensor based on microfluidic capacitance modification and radar passive detection for medical and veterinary temperature telemetry systems has been demonstrated. The fabrication process flow was detailed, and the final realized structure was tested using a VNA and probes. A $13 \mathrm{fF} /{ }^{\circ} \mathrm{C}$ of measured sensitivity was obtained. Finally, the temperature sensor was integrated with a horn antenna via 1-m-length coaxial delay line and was interrogated using the millimeter-wave FMCW radar interrogation technique. Measured results obtained show a linear sensitivity of $0.4 \mathrm{dBm} /{ }^{\circ} \mathrm{C}$ that, coupled with the miniaturized sensor volume (below $1 \mathrm{~mm}^{3}$ ), verify the potential of the presented approach for wide applicability in various "rugged" medical and wearable environments.

\section{REFERENCES}

[1] J. F. Swindells, "Precision measurement and calibration: Selected NBS papers on temperature," U.S. Government Printing Office, NBS Special Publication 300-volume 2, 1968.

[2] V. Batageli, J. Bojkovski, J. Drnovsek, and I. Pusnik, "Automation of reading liquid-in-glass thermometers," IEEE Trans. Instrum. Meas., vol. 50, no. 6, pp. 1594-1598, Dec. 2001.

[3] A. Pohl, "A review of wireless SAW sensors," IEEE Trans. Ultrason., Ferroelectr., Freq. Control, vol. 47, no. 2, pp. 317-332, Mar. 2000.

[4] A. Ruhanen, M. Hanhikorpi, F. Bertuccelli, A. Colonna, W. Malik, D. Ranasinghe, T. S. Lopez, N. Yan, and M. Tavilampi, "Sensor-enabled RFID tag handbook," Report BRIDGE Project, 2008 [Online]. Available: http://www.bridge-project.eu

[5] A. Traille, S. Bouaziz, S. Pinon, P. Pons, H. Aubert, and A. Boukabache, "A wireless passive RCS-based temperature sensor using liquid metal and microfluidics technologies," in Proc. EuMC, Oct. 10-13, 2011, pp. 45-48.

[6] M. M. Jatlaoui, F. Chebila, T. Idda, P. Pons, and H. Aubert, "Phenomenological theory and experimental characterizations of passive wireless EM pressure micro-sensor prototype," in Proc. IEEE Sensors, Nov. 2010, pp. 643-646.

[7] H. Hallil, F. Chebila, P. Menini, P. Pons, and H. Aubert, "Feasibility of wireless gas detection with an FMCW RADAR interrogation of passive RF gas sensor," in Proc. IEEE Sensors, Nov. 1-4, 2010, pp. 759-762.

[8] M. M. Jatlaoui, F. Chebila, S. Bouaziz, P. Pons, and H. Aubert, "Original identification technique of passive EM sensors using loaded transmission delay lines," in Proc. EuMC, Sep. 2010, pp. 1106-1109.

[9] H. Aubert, F. Chebila, M. Jatlaoui, T. Thai, H. Hallil, A. Traille, S. Bouaziz, A. Rifai, P. Pons, P. Menini, and M. Tentzeris, "Wireless sensing and identification of passive electromagnetic sensors based on millimetre-wave FMCW RADAR," in Procs. IEEE RFID-TA, Nov. 2012, pp. 398-403.

[10] P. Abgrall, C. Lattes, V. Conédéra, X. Dollat, S. Collin, and A. M. Gué, "A novel fabrication method of flexible and monolithic 3D microfluidic structures using lamination of SU-8 film," J. Micromech. Microeng., vol. 16, no. 1, pp. 113-121, Dec. 2005.

[11] J. Monahan, A. A. Gewirth, and R. G. Nuzzo, "A method for filling complex polymeric microfluidic devices and arrays," Anal. Chem., vol. 73, no. 13, pp. 3193-3197, May 2001. 